\title{
Analyzing Master Boot Record for Forensic Investigations
}

\author{
Ghania Al Sadi \\ Sohar University \\ General Foundation Program \\ - Computing Program \\ Sohar, University Rd, \\ 311 - Sultanate of Oman
}

\begin{abstract}
As a main knowledge, extracting information for examination to be used as evidence or even to recover lost data need a full understand of logical and physical storage media structure used to store the required information in the computer. In digital forensic analysis, Master Boot Record is captured to extract the required information of the hard disk to support the investigation process. This research is studying the MBR structure by providing an experiment of the MBR analysis.
\end{abstract}

\section{General Terms}

Master Boot Record, Booting, Forensic Investigation

\section{Keywords}

MBR, Bootstrap, Partition Table, Magic Number, Forensic Investigation

\section{INTRODUCTION}

Master Boot Record (MBR) is the most important part that exists on the first sector $(0 \mathrm{x} 00)$ in the hard drive. It holds the most required terms to boot the device and load the operating system to memory. It is a disk data structure that is created once the disk is partitioned. Disk partition is referred to logical distribution of the hard drive to multiple storage units enabling different file systems to be used on each partition. MBR exists only in partitioned storage devices and cannot be found in non-partitioned disk like floppy disk. MBR consists of the partition table of the disk and finds the bootable disk that is referred as active partitions. Moreover, it holds information related to the type and size of the partitions and the file systems used on each logical partition. In general, MBR is responsible of booting the computer using an executable code called "bootloader"[1]. MBR supports disks with sectors of 512 bytes that is the currently standard used size. Most disks supported by this partition scheme are limited with two terabyte size[2].

Since MBR is controlling booting process after the BIOS finish its job, make susceptible by malware and some potential threats. Some malicious programs may get control over booting process by altering the MBR and load malicious software to the memory. Moreover, malware like ransomware may move the MBR to different location on the disk and replace itself on the first sector of the disk. Therefore, it carries out the booting process by executing its code once the BIOS switch to the drive for booting. Generally, analyzing MBR is mandatory to extract any malicious programs that may affect the MBR since it carry out the booting process in the computer [3].

For forensic use, investigating the MBR is required since it contains all information about the recent partitions available in the system. Generally, MBR is analyzed to extract storage disks' information. If the MBR is corrupted, it will be difficult to boot the system and thus difficult to recover data from the storage disks [1]

\section{MBR STRUCTURE}

MBR is physically located in sector $0(0 \mathrm{x} 00)$; the first sector in the storage disk that is 512 bytes long. MBR structure consists of three main parts that are master boot code (bootstrap), partition table and the disk signature as shown in Figure 1. All contents of MBR are located in different locations with different number of bytes that need to be fully analyzed to extract the required information

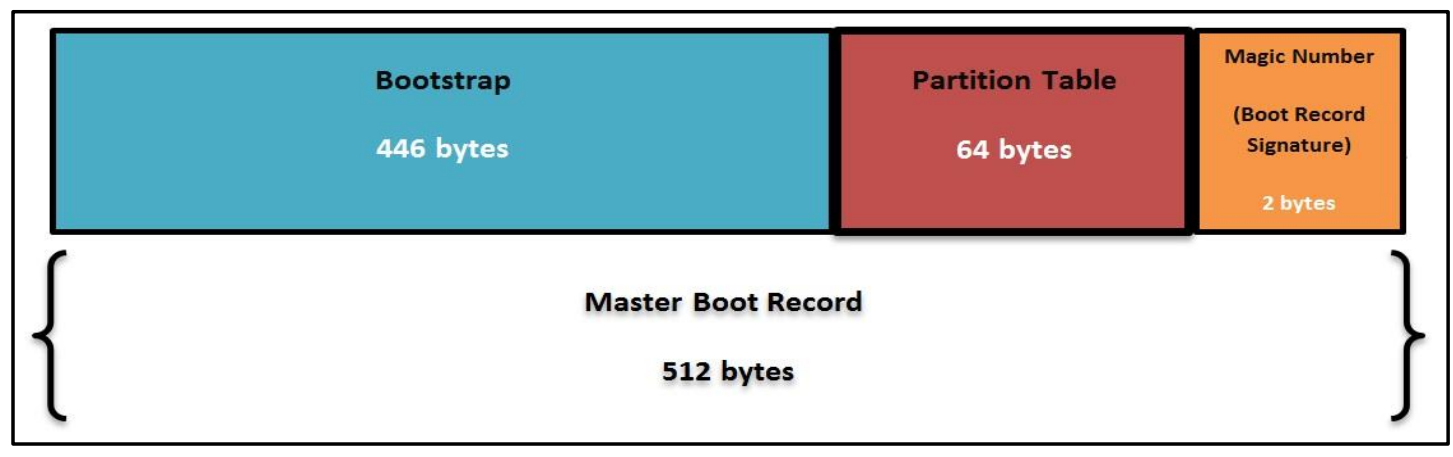

Figure 1: Master Boot Record Structure 


\subsection{Partition Table}

Partition table is a 64 bytes data structure that contains all information of the hard disk partitions. Generally, partitioning the disk to multiple partitions enables different file systems to be used on each partition. Type and location of each partition on the disk is identified by the partition table. The standard number of partitions in the table is four partitions; however the last one might be used as extended partition for other more partition. One of these partitions is marked as an active partition which is used to continue booting process in the computer. The size of the partition table is distributed equally among the four partitions. Normally, each partition table entry begins at predefined offset starting from the beginning of the sector [1].

Partition table is analyzed to find the active partition; that is used to continue booting process. Usually, MBR allows only for one active partition for booting process; however in some cases more than one active partition may exists that cause the MBR to return an error message. Active partition loads files to memory based on the type of the file system used by the partition. In case of missing the active partition, it will be difficult to recover and restore data stored in the disk [5]. Once the active partition is founded, much information may be extracted during analysis process such as the filesystem used by the partition and the size of the partition [1].

\subsection{Bootstrap}

Bootstrap is referred to as boot loader or Master Boot Code. It is an executable code that is responsible for loading the operating system to computer memory. Bootstrap code area is responsible to find the active partition by scanning the partition table and catch the first sector in this active partition. Once the active partition is scanned, a copy of the boot sector will be loaded to the memory to start controlling booting process [6]. In case that the master boot code fails to finish its functions, different types of errors will be reported by the system stating some problems with partition table or the operating system either it is not available or error in loading process. Based on the predefined MBR data structure, bootstrap has 446 byte of data structure [2].

\subsection{Boot Record Signature}

Boot Record Signature is located at the end of the MBR that can be also named as Magic Number. It is a smallest unit in MBR structure that contains only two bytes that is required by the BIOS during booting. The magic number used to report the availability of the boot loader in the hard disk. If the boot loader is located, the magic number value should be (55AA) in hexadecimal calculation [5].

\section{MBR ANALYSIS EXPERIMENT}

Generally MBR is analyzed to extract or recover required information from the hard disk. Forensic investigations analyze the MBR to find if any malicious MBR exist in the memory that my overwrite the basic or clean MBR. In case of MBR infections, more than one MBR copy may be located in the memory that may be clean or malicious MBR [7]. Therefore, it is mandatory to study the standard MBR structure to distinguish between clean and malicious MBRs. As mentioned before MBR structure consists of 512 bytes distributed among three parts. The following table specifies the specific location of each part in terms of decimal, hex and binary to simplify examining process of the MBR.

Table 1. Master Boot Record Sector Structure

\begin{tabular}{|c|c|c|c|c|}
\hline \multirow{2}{*}{\multicolumn{2}{|c|}{ MBR Structure contents }} & \multicolumn{2}{|c|}{ Offsets within MBR Sector } & \multirow{2}{*}{ Bytes (length } \\
\hline & & Decimal & Hex & \\
\hline \multicolumn{2}{|c|}{ Bootstrap Code Area } & $000-445$ & $000-1 \mathrm{BD}$ & 446 \\
\hline \multirow{4}{*}{ Partition Table } & Partition 1 & $446-461$ & $1 \mathrm{BE}-1 \mathrm{CD}$ & 16 \\
\hline & Partition 2 & $462-477$ & $1 \mathrm{CE}-1 \mathrm{DD}$ & 16 \\
\hline & Partition 3 & $478-493$ & $1 \mathrm{DE}-1 \mathrm{ED}$ & 16 \\
\hline & Partition 4 & $494-509$ & $1 \mathrm{EE}-1 \mathrm{FD}$ & 16 \\
\hline \multicolumn{2}{|c|}{ Total of Partition Table } & $446-509$ & $1 \mathrm{BE}-1 \mathrm{FD}$ & 64 \\
\hline \multicolumn{2}{|c|}{ Boot Record Signature } & $510-511$ & $1 \mathrm{FE}-1 \mathrm{FF}$ & 2 \\
\hline
\end{tabular}

As described in the table, each pattern exists in a specific offsets within the MBR that are considered as common between different machines. Therefore; finding MBR patterns in different offsets during investigation process will results in potential MBRs in the memory.

A number of tools are used to extract the required information and analyze the MBR that simplify the investigation. Generally, it is important to use an accurate tool to extract evidences from MBR during forensic analysis. In this research, Hex Workshop software is used to analyze the MBR and get accurate information. Hex Workshop it is a powerful hexadecimal tool that is more suitable for investigating sectors and partition table since it supports decimal, binary and hexadecimal data.

The research is analyzing a previously captured MBR to find Bootstrap Code Area, Active Partition and Boot Record Signature as following:

\section{- Bootstrap Code Area}

As shown in Figure 2, bootstrap code area is located in the offset $(000-445)$ which referred to $(000-1 B D)$ in hex. Since the bootstrap existing in the correct offset; then it is ready to scan the partition table to find the active partition and load the boot sector into memory. 


\begin{tabular}{|c|c|c|c|c|c|c|c|c|c|c|c|c|c|c|c|c|c|c|c|c|c|}
\hline 00 & 33 & $\mathrm{CO}$ & $8 \mathrm{E}$ & DO & $\mathrm{BC}$ & 00 & $7 \mathrm{C}$ & $8 \mathrm{E}$ & $\mathrm{CO}$ & $8 \mathrm{E}$ & D8 & $\mathrm{BE}$ & 00 & $7 \mathrm{C}$ & $\mathrm{BF}$ & 00 & 06 & B9 & 00 & 02 & \\
\hline 15 & F3 & A4 & 50 & 68 & $1 \mathrm{C}$ & 06 & $\mathrm{CB}$ & $\mathrm{FB}$ & B9 & 04 & 00 & BD & $\mathrm{BE}$ & 07 & 80 & $7 \mathrm{E}$ & 00 & 00 & $7 \mathrm{C}$ & $O B$ & $O F$ \\
\hline $2 \mathrm{~A}$ & 85 & $O E$ & 01 & 83 & C5 & 10 & E2 & F1 & $C D$ & 18 & 88 & 56 & 00 & 55 & C6 & 46 & 11 & 05 & C6 & 46 & 10 \\
\hline $3 F$ & 00 & B 4 & 41 & $\mathrm{BB}$ & $\mathrm{AA}$ & 55 & $C D$ & 13 & $5 \mathrm{D}$ & 72 & $O F$ & 81 & $\mathrm{FB}$ & 55 & $\mathrm{AA}$ & 75 & 09 & F 7 & $\mathrm{Cl}$ & 01 & 00 \\
\hline 54 & 74 & 03 & $\mathrm{FE}$ & 46 & 10 & 66 & 60 & 80 & $7 \mathrm{E}$ & 10 & 00 & 74 & 26 & 66 & 68 & 00 & 00 & 00 & 00 & 66 & $\mathrm{FF}^{\prime}$ \\
\hline 69 & 76 & 08 & 68 & 00 & 00 & 68 & 00 & $7 \mathrm{C}$ & 68 & 01 & 00 & 68 & 10 & 00 & B 4 & 42 & $8 \mathrm{~A}$ & 56 & 00 & $8 \mathrm{~B}$ & F4 \\
\hline $7 \mathrm{E}$ & $C D$ & 13 & $9 F$ & 83 & C4 & 10 & $9 E$ & $\mathrm{~EB}$ & 14 & B 8 & 01 & 02 & $B B$ & 00 & $7 \mathrm{C}$ & $8 \mathrm{~A}$ & 56 & 00 & $8 \mathrm{~A}$ & 76 & 01 \\
\hline 93 & $8 \mathrm{~A}$ & $4 \mathrm{E}$ & 02 & $8 \mathrm{~A}$ & $6 \mathrm{E}$ & 03 & $C D$ & 13 & 66 & 61 & 73 & $1 \mathrm{C}$ & $\mathrm{FE}$ & $4 \mathrm{E}$ & 11 & 75 & $O C$ & 80 & $7 \mathrm{E}$ & 00 & 80 \\
\hline 00 & $O F$ & 84 & $8 \mathrm{~A}$ & 00 & B2 & 80 & $E B$ & 84 & 55 & 32 & E4 & $8 \mathrm{~A}$ & 56 & 00 & $C D$ & 13 & 5D & $E B$ & $9 E$ & 81 & $3 \mathrm{E}$ \\
\hline$B D$ & $\mathrm{FE}$ & $7 \mathrm{D}$ & 55 & $\mathrm{AA}$ & 75 & $6 \mathrm{E}$ & FF & 76 & 00 & E8 & $8 \mathrm{D}$ & 00 & 75 & 17 & FA & BO & D1 & E6 & 64 & E8 & 83 \\
\hline D2 & 00 & BO & $D F$ & E6 & 60 & E8 & $7 \mathrm{C}$ & 00 & BO & $\mathrm{FF}$ & E6 & 64 & E8 & 75 & 00 & FB & B8 & 00 & BB & $C D$ & $1 \mathrm{~A}$ \\
\hline 00 & 66 & 23 & $\mathrm{CO}$ & 75 & $3 B$ & 66 & 81 & FB & 54 & 43 & 50 & 41 & 75 & 32 & 81 & F9 & 02 & 01 & 72 & $2 \mathrm{C}$ & 66 \\
\hline 000 & 68 & 07 & $\mathrm{BB}$ & 00 & 00 & 66 & 68 & 00 & 02 & 00 & 00 & 66 & 68 & 08 & 00 & 00 & 00 & 66 & 53 & 66 & 53 \\
\hline 11 & 66 & 55 & 66 & 68 & 00 & 00 & 00 & 00 & 66 & 68 & 00 & $7 \mathrm{C}$ & 00 & 00 & 66 & 61 & 68 & 00 & 00 & 07 & CD \\
\hline 00000126 & $1 \mathrm{~A}$ & $5 \mathrm{~A}$ & 32 & F 6 & EA & 00 & $7 \mathrm{C}$ & 00 & 00 & $C D$ & 18 & $\mathrm{~A} 0$ & B 7 & 07 & $\mathrm{~EB}$ & 08 & $\mathrm{AO}$ & B 6 & 07 & EB & 03 \\
\hline $0000013 \mathrm{~B}$ & A0 & B5 & 07 & 32 & E4 & 05 & 00 & 07 & $8 \mathrm{~B}$ & Fo & $\mathrm{AC}$ & $3 \mathrm{C}$ & 00 & 74 & 09 & $\mathrm{BB}$ & 07 & 00 & B 4 & $O E$ & $C D$ \\
\hline 00000150 & 10 & $E B$ & F2 & F4 & $\mathrm{EB}$ & FD & $2 \mathrm{~B}$ & C9 & E4 & 64 & $E B$ & 00 & 24 & 02 & E0 & F8 & 24 & 02 & C3 & 49 & $6 \mathrm{E}$ \\
\hline 00000165 & 76 & 61 & $6 \mathrm{C}$ & 69 & 64 & 20 & 70 & 61 & 72 & 74 & 69 & 74 & 69 & $6 \mathrm{~F}$ & $6 \mathrm{E}$ & 20 & 74 & 61 & 62 & $6 \mathrm{C}$ & 65 \\
\hline $017 \mathrm{~A}$ & 00 & 45 & 72 & 72 & $6 \mathrm{~F}$ & 72 & 20 & $6 \mathrm{C}$ & $6 \mathrm{~F}$ & 61 & 64 & 69 & $6 \mathrm{E}$ & 67 & 20 & $6 \mathrm{~F}$ & 70 & 65 & 72 & 61 & 74 \\
\hline $18 \mathrm{~F}$ & 69 & $6 \mathrm{E}$ & 67 & 20 & 73 & 79 & 73 & 74 & 65 & $6 \mathrm{D}$ & 00 & 4D & 69 & 73 & 73 & 69 & $6 \mathrm{E}$ & 67 & 20 & $6 \mathrm{~F}$ & 70 \\
\hline A 4 & 65 & 72 & 61 & 74 & 69 & $6 \mathrm{E}$ & 67 & 20 & 73 & 79 & 73 & 74 & 65 & $6 \mathrm{D}$ & 00 & 00 & 00 & 63 & $7 \mathrm{~B}$ & $9 \mathrm{~A}$ & E2 \\
\hline $1 \mathrm{~B} 9$ & $\mathrm{FC}$ & $O E$ & D5 & 01 & 01 & 80 & 20 & 21 & 00 & 07 & $\mathrm{FE}$ & FF & FF & 00 & 08 & 00 & 00 & 00 & DO & $5 \mathrm{C}$ & $0 c$ \\
\hline
\end{tabular}

\section{Figure 2: Bootstrap Code Area in Hex}

\section{- Partition Table:}

The partition table is investigated to find the available partitions, find the bootable and active partitions and to specify if any extended partition is available. First, to locate the partition table, we need to read between the offsets that compose the partition table staring from $1 \mathrm{BE}$ (446) and ending with 1FD (509). Using Hex Workshop, the four partition tables are located as the shown in Figure 3:

\begin{tabular}{|c|c|c|c|c|c|c|c|c|c|c|c|c|c|c|c|c|c|c|c|c|c|}
\hline p00001B9 & FC & $O E$ & D5 & 01 & 01 & 80 & 20 & 21 & 00 & 07 & $\mathrm{FE}$ & FF & EF & 00 & 08 & 00 & 00 & 00 & DO & $5 \mathrm{C}$ & $\mathrm{OC}$ \\
\hline$C E$ & & $\mathrm{EE}$ & & $\mathrm{EF}$ & & FE & & FF & 0.8 & D8 & & $O C$ & 00 & 90 & 01 & 00 & 00 & $\mathrm{EE}$ & 75 & $F F$ & $0 E$ \\
\hline E3 & & EF & $\mathrm{EB}$ & 00 & 68 & $5 \mathrm{E}$ & 00 & 00 & $B B$ & 01 & 00 & 00 & 00 & 00 & 00 & 00 & 00 & 00 & 00 & 00 & 00 \\
\hline$F^{8} 8$ & 00 & 00 & 00 & 00 & 00 & 00 & 55 & $\mathrm{AA}$ & & & & & & & & & & & & & \\
\hline
\end{tabular}

Figure 3: Partition Table Entries in Hex

Based on the result extracted from Hex Workshop tool, only one active partition is found that is partition \#1. As shown in Figure 3 , the active partition starts with the value $(80 \mathrm{~h})$ which indicate to standard active partition in the MBR sector. The other partitions are considered as non-active partition since are starting with the value (00). This indicates that only one bootable partition is available to continue booting process.
Based on the given result, only one partition will be analyzed that is the active partition. Each hexadecimal value in this active partition will be examined to extract the required information like starting and ending sectors in CHS values, the file system and the size of the partition. The partition is 16-bytes in length as shown in Figure 4 that will be examined depending on the MBR partition table structure.

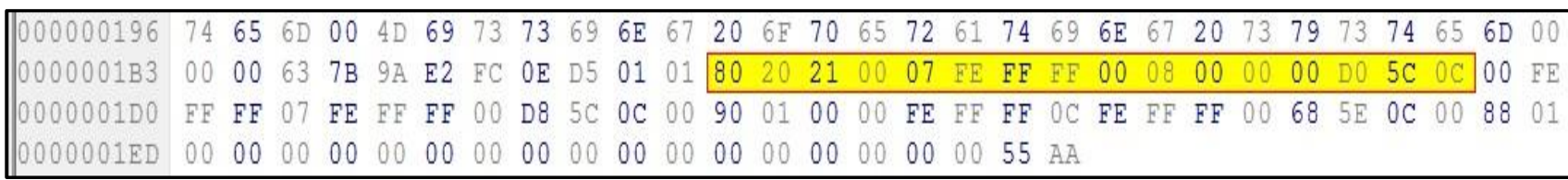

\section{Figure 4: Active Partition in Hex}

The 16-byte partition table entry is distributed among six contents of the active partition with different length of bytes. For more clarification, this structure is described in the following table that provides the results of Active partition analysis: 
Table 2. Active Partition Analyzing Results

\begin{tabular}{|c|c|c|c|}
\hline Contents & Relative Offsets & Length in bytes & Description \\
\hline $\begin{array}{c}\text { Boot } \\
\text { Indicator }\end{array}$ & $0(80)$ & 1 & $\begin{array}{l}\text { - Indication of the active partition that guide the boot loader to the } \\
\text { required partition to boot. } \\
\text { - Referred as SYSTEM partition in Windows OS. }\end{array}$ \\
\hline $\begin{array}{l}\text { Starting CHS } \\
\text { values }\end{array}$ & $\begin{array}{c}1-3 \\
(202100)\end{array}$ & 3 & $\begin{array}{l}\text { - Indicates the starting sector of the partition in Cylinder Head Sector } \\
\text { values. } \\
\text { - In hex, are read in reverse case as } \mathbf{0 0} \mathbf{2 1 2 0 .}\end{array}$ \\
\hline $\begin{array}{l}\text { Partition } \\
\text { type } \\
\text { (File System) }\end{array}$ & $4(07)$ & 1 & $\begin{array}{l}\text { - Representation of the partition's file system } \\
\text { - Also, it can be referred to as Partition ID } \\
\text { - It specifies the file system used by the partition and represents the } \\
\text { access method to the partition. } \\
\text { - The value (07) indicates to NTFS file system that is supported in } \\
\text { Microsoft Windows and Microsoft DOS. }\end{array}$ \\
\hline $\begin{array}{c}\text { Ending } \mathrm{CHS} \\
\text { values }\end{array}$ & $\begin{array}{c}5-7 \\
\text { (FE FF FF) }\end{array}$ & 3 & $\begin{array}{l}\text { - Indicates the ending sector of the partition in Cylinder Head Sector } \\
\text { values. } \\
\text { - As mentioned in the starting sector, the values are read as FF FF FE } \\
\text { in hex. }\end{array}$ \\
\hline $\begin{array}{l}\text { Starting } \\
\text { Sector }\end{array}$ & $\begin{array}{c}8-11 \\
(00080000)\end{array}$ & 4 & $\begin{array}{l}\text { - It indicates the starting sector of the active partition. } \\
\text { - Read in hex as } 00000800 . \\
\text { - Represented in decimal as } 2048\end{array}$ \\
\hline Partition Size & $\begin{array}{c}12-15 \\
(00 \mathrm{DO} 5 \mathrm{C} \mathrm{OC})\end{array}$ & 4 & $\begin{array}{l}\text { - Represents partition size in sectors. } \\
\text { - It is read as 0C 5C D0 } 00 \text { in hex. } \\
\text { - The size in sectors is } \mathbf{2 0 7 4 0 9 1 5 2 ~ s e c t o r s ~ ( d e c i m a l ) . ~} \\
\text { - The size in bytes is: } \\
\text { - } \mathbf{1 0 6 1 9 3 4 8 5 8 2 4} \text { Bytes = } \mathbf{1 0 1 2 7 4} \mathrm{MiB}=\mathbf{9 8 . 9} \mathrm{GiB}\end{array}$ \\
\hline
\end{tabular}

As mentioned in the above table, the used file system is NTFS. The partition start at sector 2048 and the size of the partition is 98.9 Gigabytes.

\section{- Boot Record Signature}

Locating the Boot record signature or the magic number requires searching in the offsets $1 \mathrm{FE}$ and $1 \mathrm{FF}$ that represent the 2-byte value as (55AA) in hex as shown in Figure 5.

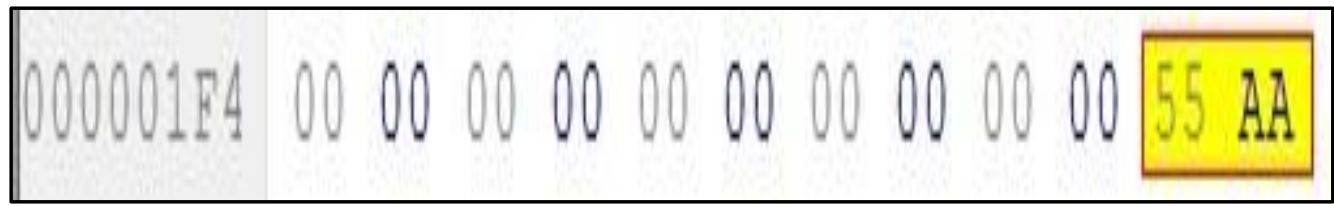

Figure 4: Boot Record Signature in Hex

Most required information is extracted easily from the MBR since it has a standard structure followed by most computer machines. In this research, only one active partition is analyzed to clarify the basic structure of the MBR and to extract the required disk information for forensic investigations.

\section{CONCLUSION}

MBR is an essential part in the computer that is physically located in the first sector of the disk and controls the booting process in the computer. As presented in this research, all MBR patterns are located in standard locations in the first sector. Therefore, studying its structure is required to make sure that the computer is not infected by malicious programs like malware that may replace the MBR and load malicious software to the computer memory during booting process. Moreover, this helps forensic investigators to extract disk partition details like the file system type used by the portion and the disk partition size. Further research might be required in the future to study the vulnerabilities exploited by malicious programs to infect the MBR and get control over booting process.

\section{REFERENCES}

[1] R. G. Minnich, "Operating System," 2004.

[2] Microsoft, "Windows support for hard disks that are larger than 2 TB," 2013. [Online]. Available: http://support.microsoft.com/kb/2581408\#appliesto.

[3] P. ARNTZ, "Meet the Master Boot Record," 2014 [Online].Available:https://blog.malwarebytes.org/securit y-threat/2014/09/meet-the-master-boot-record/. 
[4] M. TechNet, "Master Boot Record," 2011. [Online]. Available:http://technet.microsoft.com/enus/library/cc97 6786.aspx.

[5] M. TechNet, "Disk Concepts and Troubleshooting," 2011.[Online].Available:http://technet.microsoft.com/enus/library/cc977219.aspx.
[6] J. Gu and W. Ji, "A secure bootstrap based on trusted computing," Proc. - 2009 Int. Conf. New Trends Inf. Serv. Sci. NISS 2009, no. 3, pp. 502-504, 2009.

[7] R. McKemmish, "What is forensic computing?," Trends Issues Crime Crim. Justice, vol. 118, no. 118, pp. 1-6, 1999. 